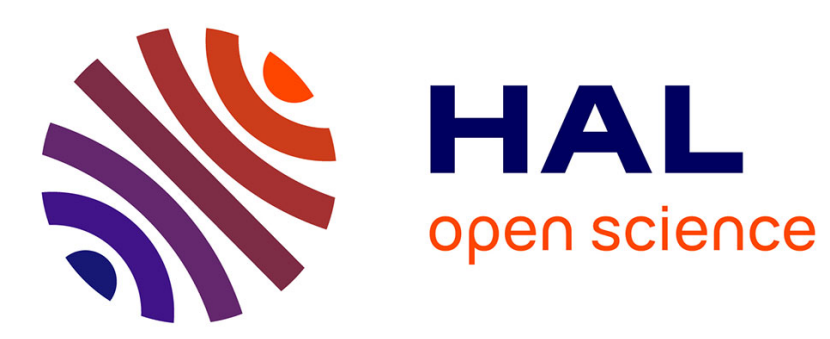

\title{
Société urbaine et désir de mission: les ressorts de la mobilité missionnaire jésuite à Milan au début du XVIIe siècle
}

Aliocha Maldavsky

\section{- To cite this version:}

Aliocha Maldavsky. Société urbaine et désir de mission: les ressorts de la mobilité missionnaire jésuite à Milan au début du XVIIe siècle. Revue d'Histoire Moderne et Contemporaine, 2009, juilletseptembre (56-3), pp.7-32. 10.3917/rhmc.563.0007 . hal-01402524

\section{HAL Id: hal-01402524 \\ https://hal.parisnanterre.fr/hal-01402524}

Submitted on 4 Jan 2018

HAL is a multi-disciplinary open access archive for the deposit and dissemination of scientific research documents, whether they are published or not. The documents may come from teaching and research institutions in France or abroad, or from public or private research centers.
L'archive ouverte pluridisciplinaire HAL, est destinée au dépôt et à la diffusion de documents scientifiques de niveau recherche, publiés ou non, émanant des établissements d'enseignement et de recherche français ou étrangers, des laboratoires publics ou privés. 


\title{
Société urbaine et désir de mission: les ressorts de la mobilité missionnaire jésuite à Milan au début du XVII ${ }^{\mathrm{e}}$ siècle
}

\begin{abstract}
Aliocha MALDAVSKY
Au début du XVII ${ }^{\mathrm{e}}$ siècle, les découvertes américaines et les voyages en Orient ont produit un monde où les hommes, les images et les idées circulent: une «mondialisation ibérique» ${ }^{1}$. On peut aller à la rencontre du vaste monde dans les habits d'un marchand, d'un administrateur, d'un soldat ou d'un homme d'Église. Des flottes régulières quittent Lisbonne, Séville et Cadix, faisant du voyage océanique une expérience certes rare, mais bien plus fréquente qu'un siècle plus tôt. Au même moment, en Europe, se développent de nouvelles pratiques dévotionnelles dans le cadre de la réforme catholique. Au-delà de la recherche de son salut personnel, le dévot "est aussi un chrétien qui se sent investi d'une véritable vocation missionnaire à l'égard des autres $»^{2}$. La rencontre entre l' «Europe des dévots" et la mobilité inscrite dans cette "mondialisation" contribue à rendre possible l'entreprise d'expansion du christianisme dans le cadre des missions menées par les ordres missionnaires.

L'Église catholique et ses diverses institutions, les monarchies à la tête des empires, sont les médiatrices de cette rencontre entre la société urbaine européenne et la conversion des "gentils" d'Orient ou d'Occident. C'est dans les villes européennes, où se développent de nouvelles pratiques dévotionnelles et les ordres religieux qui les promeuvent, comme la Compagnie de Jésus, que l'on croise les missionnaires qui vont convertir les païens. Les Indipetae, lettres de demande pour partir aux Indes rédigées par les jésuites, expriment la vocation de ces religieux européens pour la mission. La société modelée par la réforme catholique non seulement approuve l'effort missionnaire, mais y participe avec sa jeunesse, pendant toute la période moderne. C'est l'hypothèse qui guide ici la lecture des lettres rédigées par les jésuites milanais et des indices de la
\end{abstract}

1. Serge GRUZINSKI, Les quatre parties du monde. Histoire d'une mondialisation, Paris, La Martinière, 2004, réed. Points-Seuil, 2006.

2. Louis CHÂTELLIER, L'Europe des dévots, Paris, Aubier-Flammarion, 1987, p. 34. 
collaboration, au début du XVII ${ }^{\mathrm{e}}$ siècle, entre les missionnaires venus notamment d'Amérique, et les ecclésiastiques et les dévots de cette deuxième Rome, dominée par les Habsbourg d'Espagne.

Milan est une étape pour les procureurs jésuites des Indes orientales et occidentales qui vont à Lisbonne, Madrid puis Rome et recrutent des missionnaires. La ville, pièce maîtresse dans l'échiquier européen des Habsbourg, située sur le chemin des Flandres, est moins tournée vers le large que vers le nord de l'Europe. Au début du XVII ${ }^{\mathrm{e}}$ siècle, le Milanais connaît les turbulences de la politique internationale : la trêve de Douze ans n'est signée qu'en 1609, les visées de la France et de la Savoie sur Montferrat, interrompues par l'assassinat d'Henri IV, refont surface en 1613, cinq ans avant le début de la guerre de Trente $a_{n}{ }^{3}$. Sans doute moins abreuvée de récits de première main que Séville, Lisbonne, Gênes, ou même Paris avec la Nouvelle France ${ }^{4}$, Milan pourrait donc être taxée d'indifférence à la mission lointaine. Pourtant, la capitale de la Lombardie est un lieu de rencontre privilégié entre la société urbaine italienne et les militaires et administrateurs espagnols de la Monarchie catholique, dont les carrières sont internationales. Le Milanais appartient à l'horizon impérial hispanique auquel participent l'Amérique, les Philippines et les Indes portugaises pendant la période d'union des couronnes. Proche de Venise, grand centre éditorial de l'époque abreuvant l'Italie et l'Europe de publications sur le vaste monde, la ville est le siège archiépiscopal de Charles puis de Frédéric Borromée, le laboratoire de la réforme catholique dans la seconde moitié du XVI ${ }^{\mathrm{e}}$ siècle $^{5}$.

Cette ouverture sur l'empire ibérique et l'ancrage milanais dans le renouveau du catholicisme européen s'expriment dans les lettres Indipetae écrites par les jeunes jésuites de la ville, qui manifestent concrètement l'horizon missionnaire de la société milanaise au début du XVII ${ }^{\mathrm{e}}$ siècle. On y lit les espoirs de voyage et de salut dans la mission lointaine d'une jeunesse citadine, issue du patriciat urbain. On y perçoit les amitiés nouées dans la perspective du projet missionnaire. On y cherche les sources d'inspiration de tels désirs. Au-delà de la jeunesse de la ville, la perspective de la mission lointaine touche plus largement la société dévote citadine à travers le rôle des institutions religieuses. Car si la Compagnie de Jésus suscite des vocations grâce à sa propagande missionnaire, elle va également à la rencontre d'un "désir des Indes" qui se construit en-dehors de ses murs, parmi les laïcs, au sein de familles et de réseaux de sociabilité religieuse ${ }^{6}$. Les rédacteurs des lettres Indipetae se situent à la frontière

3. Gianvittorio SignOROTTO, Milano spagnola. Guerra, istituzioni, uomini di governo, Milan, Sansoni, 2001, p. 19-20; Antonio Domínguez OrTIZ, Historia de España, tome 3 : El Antiguo Régimen: los Reyes católicos y los Austrias, Madrid, Alianza editorial, 1996, p. 302-305. 2003.

4. Dominique DESLANDRES, Croire et faire croire. Les missions françaises au XVII siècle, Paris, Fayard,

5. G. SignOROTTO, "Milano sacra. Organizzazione del culto e consenso tra XVI e XVIII secolo", in Franco Della Peruta, Roberto Leydi, Angelo Stella (éd.), Milano e il suo teritorio, Milan, Silvana, 1985, p. 581-629.

6. D. DESLANDRES, Croire et faire croire, op. cit., p. 74-86 et 235-240. 
entre le monde des laïcs et celui des religieux. Cette construction se nourrit d'informations, d'une conception active de la vie religieuse par les laïcs ${ }^{7}$, mais aussi de mythes.

\section{LES INDIPETAE MILANAISES AU DÉBUT DU XVII ${ }^{\mathrm{e}}$ SIÈCLE}

Les archives romaines de la Compagnie de Jésus conservent un ensemble d'environ 14000 lettres manuscrites, dont la rédaction s'échelonne entre les années 1570 et la suppression de l'Ordre (1773), provenant des provinces jésuites d'Europe. Les aspirants à la mission formulent leur désir et leurs motivations personnelles dans une lettre de demande des Indes, dite Indipeta, qu'ils adressent au général à Rome, selon une idée d'Ignace de Loyola ${ }^{8}$. À partir du tournant des années 1580, lorsque les effectifs de l'ordre dépassent les 5000 personnes et que leur dispersion est mondiale, la rédaction des Indipetae et leur archivage correspondent à une procédure administrative d'engagement. Tout partant doit signifier la liberté d'un choix dont la lettre constitue une preuve juridique, protégeant l'Ordre des attaques de familles parfois peu disposées à laisser partir leur enfant définitivement. Le soin accordé à la conservation des lettres indique la création d'une véritable administration des vocations et explique le nombre considérable de lettres archivées ${ }^{9}$.

L'extraction des lettres des jésuites de la province de Milan, tous domiciles confondus, aboutit à un total de 68 noms, pour près de 160 documents rédigés entre 1603 et $1613^{10}$. Alors que les lettres italiennes datées ne sont que 23 en 1600, par la suite elles dépassent la centaine, avec par exemple 176 lettres en 1608, dont 42 milanaises.

À l'échelle de la province de Milan, si on considère l'année 1606, pour laquelle on dispose d'un catalogue triennal, on trouve 101 lettres Indipetae datées, dont seize milanaises. La province compte alors 335 membres répartis

7. Jean-Pierre GuTTON, Dévots et socièté au XVII siècle. Construire le ciel sur la terre, Paris, Belin, 2004.

8. Cf. les articles d'Anna Rita CAPOCCIA, Charlotte DE CASTELnAU-L'EstoIle, Pierre-Antoine FABRE et Aliocha MALDAVSKY in P.-A. FABRE, Bernard VINCENT (éd.), Notre lieu est le monde. Missions religieuses dans le monde ibérique à l'époque moderne, Rome, École française de Rome, 2007; Giovanni PIZZORUsso, "Le choix indifférent. Mentalités et attentes des jésuites aspirants missionnaires dans l'Amérique française au XVII ${ }^{\mathrm{e}}$ siècle", Mélanges de l'École Française de Rome. Italie-Méditerranée, 10-2, 1997, p. 881-894; Alessandro GUERRA, «Per un'archeologia della strategia missionaria dei Gesuiti: Le Indipetae e il sacrificio nella "la vigna del signore" ", Archivio italiano per la storia della pietà, XIII, 2000, p. 109-191; Gian Carlo Roscioni, Il desiderio delle Indie. Storie, sogni e fughe di giovani gesuiti italiani, Turin, Einaudi, 2001.

9. A. MALDAVSKY, "Administrer les vocations missionnaires. Les Indipetae et l'organisation des expéditions de missionnaires aux Indes Occidentales au début du XVII ${ }^{\mathrm{e}}$ siècle", in P.-A. FABRE et B. VINCENT (éd.), Notre lieu est le monde..., op. cit., p. 45-70.

10. Le dépouillement de ce fonds a été mené, avec P.-A. FABRE, dans le cadre d'un projet ACI sur le fonds Indipetae, au sein du Groupe de recherches sur les missions ibériques modernes (EHESS-CARE). Archivum Romanum Societatis Iesu (désormais ARSI), Fondo Gesuitico (désormais FG) 732 (sans date), FG 733 (1589-1606), FG634 (1607-1615); ARSI, Ital. 173, "Missiones et servitium peste infectorum petentes". 
dans quinze établissements, situés de Crémone jusqu'à Nice, en passant par Gênes et jusqu'à la Corse, avec une résidence à Bastia ${ }^{11}$. En proportion, les rédacteurs d'Indipetae dans la province de Milan sont peu nombreux, d'autant que certains écrivent beaucoup, comme Giovanni Rho, qui envoie 10 lettres entre 1607 et 1609, ou Giovanni Giacomo Pasquale, avec 26 lettres entre 1604 et 1613 .

Ces preuves de ténacité et de motivation ne semblent pourtant pas soutenues par une maîtrise très approfondie des informations sur les Indes et la mission.

\section{L'HORIZON DE LA MISSION AUX INDES : ENTRE CONSCIENCE ET MÉCONNAISSANCE}

Si la destination souhaitée est précise, la majorité demandant les Indes orientales, rares sont les détails sur les lieux où les rédacteurs désirent aller. Cette omission s'explique en partie par la nature même des Indipetae, l'expression de la vocation pour la mission exigeant l'indifférence, preuve de la disponibilité spirituelle et de l'obéissance due aux supérieurs, seuls habilités à décider de la destination. C'est donc souvent au détour d'un argument en faveur de la candidature qu'on perçoit la possession d'informations assez précises sur les qualités requises ou sur les dangers.

Les rédacteurs ont conscience de l'importance de certains savoirs en fonction des lieux demandés. En 1603, Antonio Antoniotti, âgé de 35 ans et doué en mathématiques, demande la Chine. On sait qu'en 1618 le choix de Nicolas Trigault se porta sur Giacomo Rho, recommandé par le cardinal Bellarmin pour ses connaissances en mathématiques, requises pour séduire la cour impériale chinoise depuis le travail de Matteo Ricci, mort en 1610. Toujours en 1603, Cesare Antora, âgé de 36 ans, préfère la Chine "parce que, ayant exercé dans les missions du Piémont parmi les hérétiques", il se sent une inclination particulière à "expliquer et défendre la foi auprès de personnes de bon intellect». Il connaît la hiérarchie des peuples qui circule dans la littérature géographique, comme c'est le cas dans l'œuvre de José de Acosta ou dans l'ouvrage de Giovanni Botero, Le relazioni universali. Par cette préférence, il exprime sa faible attirance pour la conversion des Indiens du Brésil ou du Paraguay. Inversement, Giovanni Giacomo Pasquale écrit en 1608 qu'il veut aller dans les «lieux les plus déserts et les plus barbares ${ }^{12}$. La hiérarchie des missions semble très nettement intégrée aux choix exprimés.

La question du martyre montre une certaine connaissance du terrain. En 1603, Giuseppe Daddei écrit que sa demande pour le Pérou suit une tentative malheureuse pour les Indes orientales, qu'il considère comme plus dangereuses. En 1605, Andrea Bianco insiste sur son désir du martyre et préfère le 
Japon parce que c'est un «lieu adapté au martyre». En 1611, le désir de martyre de Ludovico Pozzo semble assez indifférent au lieu puisqu'il demande en vrac: l'Inde, le Mexique, le Japon, le Brésil, ou l'Angleterre ${ }^{13}$. Il ne faut cependant pas exagérer le désir de martyre de la part des rédacteurs. Lorsqu'ils écrivent qu'ils veulent mourir aux Indes, c'est aussi sans doute qu'ils ont une pleine conscience du caractère définitif d'un tel départ.

L'apprentissage des langues revient aussi très souvent dans les lettres. En 1605, Giovanni Battista Sacco désire apprendre la langue du pays où il ira, et voudrait partir rapidement, conscient qu'il vaut mieux être jeune pour apprendre une langue étrangère. En 1609, Giovanni Pietro Patarini évoque Niccoló Spinola, procureur du Japon en Italie, et explique qu'il a des facilités pour les langues. En avril 1609, Giovanni Rho évoque la "mission» de Goa et l'apprentissage des langues ${ }^{14}$.

Les rédacteurs connaissent les conditions globales de la mission, ou du moins les arguments mis en avant par les jésuites eux-mêmes pour recruter des candidats. En 1606, Fegnoli et Cignardi évoquent ensemble un désir ancien qui s'est développé depuis les nouvelles de l'ouverture à l'Évangile de la Guinée africaine. Bien informés des circuits et des itinéraires, ils pensent que c'est le bon moment pour partir à Lisbonne puis à Goa. En 1609, Giovanni Giacomo Pasquale déclare au général que, s'il ne l'envoie pas, il aura sur la conscience toutes les âmes non converties aux Indes, étant donné la pénurie d'ouvriers que déplorent, selon lui, ces provinces. Vincenzo Rignoli pense en 1609 que «les Maures prêchent leur infâme secte dans de nouveaux pays de l'Inde et on la reçoit à la place de l'Évangile ${ }^{15}$. Cette allusion au contexte général de lutte contre l'expansion de l'islam montre à la fois une connaissance et une méconnaissance du terrain, étant donné que les pays de l'Inde, comme il dit, ne sont nouveaux que pour les Européens à cette époque, les musulmans y étant entrés depuis longtemps.

Savants et ignorants à la fois, les rédacteurs des Indipetae, sont renseignés aussi bien sur des aspects pratiques de la mission que sur son contexte géopolitique, informations sans doute puisées aussi bien dans le récit des missionnaires de retour en Europe que dans leurs écrits. Les Indipetae montrent que les individus constituent un vecteur essentiel de la connaissance que les rédacteurs ont des terres de mission. Les supérieurs, qu'il s'agisse des procureurs ou des membres de la province d'origine, jouent un rôle essentiel dans la rédaction des lettres et dans la transmission des informations ${ }^{16}$. Les lettres milanaises citent tous les horizons, montrant à quel point Milan est un lieu de passage pour les procureurs. Les Indes occidentales apparaissent à l'occasion du passage de Diego de Torres Bollo en 1603, d'Alonso Messia en 1608, procureurs du Pérou,

13. FG733 f. 221-221v.; FG733 f. 331-331v.; FG734 f. 367rv.

14. FG733 f.318-318v; FG734 f. 295-295v; FG734 f. 292-292v.

15. FG734 f. 257-257v; FG734 f. 294-294v.

16. A. MALDAVSKY, "Administrer les vocations...", art. cit. 
ou de Martin de Funes dans les années 1608 et 1609, ancien professeur de théologie à Milan, parti en 1603 puis revenu de la Nouvelle Grenade. Les Indes orientales apparaissent à l'occasion de rencontres avec un procureur de Pékin en 1608, des Philippines en 1603 (le Père Chirino), puis Nicoló Spinola, procureur du Japon cité par de très nombreux candidats ${ }^{17}$.

Est-ce à dire que dans les collèges de la Compagnie de Jésus les livres ne jouent aucun rôle dans cette éducation à la géographie et à l'histoire des terres de mission? L'enseignement de l'histoire et de la géographie s'inscrit dans les humanités, s'appuyant sur les œuvres des auteurs de l'Antiquité, et rarement sur les plus récentes publications. Mais même si le Ratio Studiorum ne le prescrit pas, les professeurs enseignent l'histoire des découvertes américaines et des explorations asiatiques, ainsi que la géographie du Nouveau Monde ${ }^{18}$. Les Avvisi, ou Lettres indiennes, publiées régulièrement par la Compagnie de Jésus depuis les années 1550, sont lues dans les réfectoires des collèges et promènent les jésuites d'Europe dans les Indes d'orient et d'occident: le Japon, Malacca, Goa, Ormuz, l'Ethiopie, autant de lieux qui résonnent aux oreilles des étudiants européens ${ }^{19}$. Le savoir transmis dans le cadre de l'oralité et diffusé dans les collèges diffère du savoir scolaire, destiné à former de futurs gouvernants ${ }^{20}$.

Les publications de la Compagnie de Jésus promouvant ses activités missionnaires dans les Indes orientales et occidentales ciblaient un public plus large que celui des collèges. L'exemple d'un ouvrage rédigé par un des procureurs rencontrés par les rédacteurs des Indipetae de ce début du XVII ${ }^{\mathrm{e}}$ siècle en Italie, Diego de Torres Bollo, manifeste les enjeux de cette littérature. De passage en Europe en tant que procureur de la province péruvienne, Diego de Torres Bollo publia en 1603, en italien et à Rome, un texte d'une soixantaine de pages intitulé Relatione breve del P. Diego de Torres della Compagnia di Giesu. Très rapidement réédité en italien, à Venise en 1604, puis traduit et publié dans les principaux centres d'impression européens, il connut une traduction polonaise dès 1603 et arriva en français dans la bibliothèque d'Henri IV en $1604^{21}$. Considéré comme le premier document sur les missions jésuites du Paraguay,

17. Les procureurs de provinces de missions sont largement cités dans les lettres: de Pékin (FG734 f. 169rv), du Japon, Nicoló Spinola (FG733 f. 310-310v; FG734 f. 221-221v; FG734 f. 290-290v; FG734 f. 217-217v; FG734 f. 248-248v, FG734 f. 294-294v), du Pérou, Diego de Torres Bollo (FG733 f. $242-$ 242v; FG733 f. 221-221v); Alonso Messia (FG734 f. 174rv; FG734 f. 183rv; FG734 f. 057-57v; FG734 f. 179rv), Martin de Funes (FG734 f. 044 -44v; FG734 f. 049-49v; FG734 f. 047-47v; FG733 f. 248 248v; FG734 f.055-55v). 470 .

18. François DE DAINVILle, L'éducation des jésuites (XVIT-XVIIT siècle), Paris, Minuit, 1978, p. 427-

19. Avvisi Particolari delle Indie di Portugallo. Nouamente hauuti questo Anno del 1555 da li R.padri della Compagnia di Iesu, Romae apud Antonium Bladum Impressorem Cameralem, 1556.

20. Daniel Nordman, "La géographie des jésuites", in Luce GIARD (éd.), Les jésuites à la Renaissance. Système éducatif et production du savoir, Paris, PUF, 1995, p. 221-236.

21. Antonio Palau y DULCET, Manual del librero hispanoamericano, t. XXIII, Barcelone-Oxford, Tecla-Tovar, 1971, p. 420-421; Carlos Sommervogel, Bibliothèque de la Compagnie de fésus, t. 8, Bruxelles-Paris, Schepens-Picard, 1898, c. 132-134; Robert STREIT, Bibliotheca missionum, t. II, RomeFreiburg-Vienne, Herder, 1959-1961, c. 1349. 
ce recueil de lettres de missionnaires du Pérou et du Paraguay vante les mérites de la mission naissante. Il fait donc la publicité de la mission aux frontières, alors que celle-ci est loin de constituer la principale activité des jésuites de la province péruvienne. Conscient de la préférence des jésuites d'Europe pour les Indes orientales et de la fascination qu'exerce l'Asie sur l'Europe, l'auteur n'hésite pas à déformer la réalité des activités de l'ordre en Amérique pour recruter des religieux motivés pour la conversion des Indiens ${ }^{22}$.

La propagande jésuite pour la mission n'est pas la seule littérature susceptible de nourrir le désir des Indes parmi les jésuites milanais, ou plus largement italiens. Une abondante littérature sur les terres lointaines éditée en langues vernaculaires ou en latin garnit sans doute les rayons des bibliothèques familiales des rédacteurs milanais d'Indipetae.

\section{LA CONNAISSANCE PAR LES LIVRES}

Il n'est évidemment pas simple d'évaluer l'impact de la littérature concernant les terres de mission sur les sociétés européennes. Au tournant du $\mathrm{XVII}^{\mathrm{e}}$ siècle, l'amateur de livres dispose de sources directes, en langue vernaculaire ou en latin, et d'une production intellectuelle d'auteurs européens sur les découvertes et l'élargissement de l'horizon géographique de l'Europe à la Renaissance. Ces écrits construisent les représentations que les Européens sont susceptibles de partager et qui varient selon le lieu où ils vivent, la confession qu'ils professent, le camp où ils se trouvent dans les rivalités politiques. Ceux qui n'écrivent pas de livres possèdent parfois des bibliothèques, même si cellesci ne disent pas ce qu'en pensent leurs propriétaires ni même s'ils ont lu les ouvrages. De ce point de vue, les lettres Indipetae semblent une source unique.

\section{La bibliographie disponible au début du XVII siècle}

Territoire dominé par les Habsbourg d'Espagne, le Milanais fait partie du vaste empire qui, au début du XVII ${ }^{\mathrm{e}}$ siècle, se situe dans la période de sa plus large extension, du fait de l'union des couronnes hispaniques et portugaise depuis 1580. La place des Milanais et des Italiens s'inscrit dans le contexte de liens étroits qu'entretient Milan avec la capitale de la monarchie et les milieux du pouvoir central. Cette proximité explique en partie la circulation, en Italie du nord et du sud, d'objets et des livres concernant les Indes en général.

Parmi les sources de l'homme lettré vivant dans la péninsule italienne au début du XVII ${ }^{\mathrm{e}}$ siècle sur les continents américain, asiatique et africain, les grandes synthèses géographiques, héritières de la tradition ptoléméenne,

22. A. MALDAVSKY, "Quitter l'Europe pour l'Amérique. Mode d'emploi d'une quête missionnaire», in Christian Buchet (éd.), La Mer, la France et l'Amérique Latine, Paris, Presses de l'université ParisSorbonne, 2006, p. 147-166. 
régulièrement mises à jour et renouvelées, côtoient les récits des grands noms des découvertes, les lettres de missionnaires ou les grandes synthèses comme celles de Botero, de Boemus ou d'Acosta. Pour les premiers temps des découvertes américaines, nombreux sont les explorateurs et voyageurs dont les écrits sont immédiatement diffusés en Italie, faisant l'objet de compilations et de réélaborations. Colomb, Vespucci, Pigafetta, pour ne parler que des plus célèbres voyageurs, mais aussi des compilateurs et médiateurs comme Pierre Martyr, B. Bordone, avec son Isolario (Venise, 1534), ou le célèbre Giambattista Ramusio, dont on retrouve l'écho chez les humanistes italiens comme P. Giovio (Historiarum sui temporis, Florence 1550-52), Pietro Bembo (Rerum Venetarum historiae, Venise, 1551), ou Guicciardini, dans sa Storia d'Italia ${ }^{23}$.

Ainsi, dès les années 1530, voient le jour les premières traductions en italien des ouvrages des conquérants: Ramusio publie la Relation du Michoacán de Nuño de Guzmán en 1530 et fait connaître Cortés, Oviedo (traduit en 1556 dans Delle navigationi e viaggi, Venise). Agostino di Cravaliz traduit López de Gomara en 1556 à Rome et Cieza de León (traduit en 1555 par Cravaliz et publié à Rome, puis à Venise en 1557 par un anonyme). Alfonso de Ulloa, militaire et lettré espagnol installé à Venise, y fait connaître des écrits de Fernand Colomb et d'Agustin de Zarate (1563, Venise) mais aussi de Portugais comme Fernao Lopes de Castanheda (Historia delle Indie Orientali, Venise, 1577) et Joao Barros (L'Asia, Venise, 1561). Dès les années 1530, on lit la correspondance de missionnaires franciscains ${ }^{24}$, puis les récits des missionnaires jésuites prennent ensuite le relais, avec la publication de recueils de lettres de missionnaires en Orient et au Brésil dans les années 1550 et 1560 . À partir des années 1580, sous l'impulsion de Claudio Aquaviva, l'édition de recueils de lettres annuelles en latin est systématique, complétées par l'œuvre du jésuite Giovanni Pietro Maffei, Historiarum Indicarum, Florence, 1588.

La domination de l'Espagne sur l'Italie explique qu'on ne dispose pas des œuvres de Las Casas en italien avant 1626, date de la publication dans la Venise indépendante de la Brevísima relación de la destrucción de las Indias, alors que Juan Gines de Sepúlveda est diffusé en latin à Rome dès $1550^{25}$. Les amateurs peuvent néanmoins lire Joannes Boemus, Gli costumi e la usanze dell'Indie occidentali, overo Mondo Nuovo, édité à Venise en 1560, et la Historia del Mondo Nuovo, de Girolamo Benzoni, publiée à Venise en 1572, qui est une expérience directe du voyage, à l'instar de l'œuvre du jésuite José de Acosta, dont la Historia Natural y moral de las Indias est éditée également à Venise en 1596. À Milan, Le Relationi universali, de Giovanni Botero, conseiller de Federico Borromeo, sont disponibles à partir des années 1592-1593, puis rééditées.

23. Rosario Romeo, Le scoperte americane nella coscenza italiana del Cinquecento, Rome-Bari, Laterza, 1989 , p. 5-26.

24. Maria Matilde BENZONI, La cultura italiana e il Messico. Storia di un'immagine da Temistitan all'Indipendenza (1519-1821), Milan, Unicopli, 2004, p. 37-43.

25. R. ROMEO, Le scoperte americane, op. cit., p. 86. 


\section{Le savoir sur les Indes}

Sur les traces de La Invención de América d'Edmundo O'Gorman (Mexico, 1958), les travaux prolongent la longue tradition d'étude de l'impact des découvertes américaines sur la culture européenne de la Renaissance aux Lumières $^{26}$. L'importance des sources espagnoles dont disposent les Italiens au $\mathrm{XVI}^{\mathrm{e}}$ siècle se traduit par une information marquée par l'apologie de la conquête, dans un cadre de domination de l'Espagne sur l'Italie. L'Italie participe ainsi à la formation d'une légende rose, ou blanche, de la conquête du Nouveau Monde, dans une exaltation de l'idéologie impériale espagnole. Seule l'œuvre de G. Benzoni critique ouvertement l'attitude des Espagnols, sans grand succès éditorial en Italie, malgré son destin polémique dans le monde protestant $^{27}$.

Sur le plan religieux, la conquête constitue le moyen de la mission universelle de l'Église, et les descriptions des us et coutumes des populations américaines, qui mettent l'accent, dans la seconde moitié du $\mathrm{XVI}^{\mathrm{e}}$ siècle, sur l'intervention du diable, confortent les auteurs dans l'idéologie tridentine de la nécessité d'un contrôle étroit des populations par l'intermédiaire de règles liturgiques et sacramentaires. La classification des peuples barbares de José de Acosta, utilisée aussi par Giovanni Botero et reprise par Antonio Possevino dans la Bibliotheca Selecta, va dans le sens d'une «conception statique de la vie civile, comme garantie d'ordre et de sécurité", selon des lois établies dans le cadre d'un État, en vigueur en Italie et héritée de la littérature utopique de la Renaissance $^{28}$. La présentation par Botero des grandes civilisations amérindiennes, des Incas et des Aztèques, comme un des degrés vers la vie civile, rassure les contemporains sur leurs critères d'évaluation des peuples du monde. À l'inverse, les "sauvages" et les barbares de la troisième catégorie, dont on expose la cruauté et l'inhumanité, à travers notamment l'anthropophagie, représentent une altérité absolue pour les Italiens pétris à la fois des idées de la Renaissance et de l'idéologie tridentine: les conceptions relativistes d'un Montaigne n'ont pas cours dans l'Italie de la fin du XVI ${ }^{\mathrm{e}}$ siècle.

L'œuvre de Giovanni Botero (1544-1617), jésuite pendant vingt ans, de 1560 à 1580, collaborateur de Charles Borromée, puis précepteur et conseiller de son neveu Federico, constitue ainsi un vecteur fondamental de cette anthropologie des peuples du monde et de la connaissance des pays lointains par le public européen, italien et milanais avec ses Relationi Universali et

26. Voir aussi Adriano ProsPerI, Wolfgang REINHARD (éd.), Il Nuovo Mondo nella coscienza italiana e tedesca del Cinquecento, Bologne, Il Mulino, 1992; William M. HAMLIN, The Image of America in Montaigne, Spenser, and Shakespeare: Renaissance Ethnography and Literary Reflection, Basingstoke, MacMillan, 1995 ou encore Jean-Marc BESSE, Les grandeurs de la Terre: aspects du savoir géographique à la Renaissance, Lyon, ENS éditions, 2003.

27. M. M. BENZONI, La cultura italiana, op. cit., p. 91.

28. Ibidem, p. 95; R. RomeO, Le scoperte americane, op. cit., p. 107; Anthony PAGDEN, The Fall of Natural Man. The American Indian and the Origins of Comparative Ethnology, Cambridge, Cambridge University Press, 1982. 
d'autres œuvres en latin et en italien ${ }^{29}$. Dans une œuvre publiée à Rome en 1599, Dell'Uffitio del Cardinale, Botero dresse un bilan de l'évangélisation dans le monde et conclut à une large diffusion de la foi catholique, surtout en Amérique, alors que dans les autres continents, il constate une extension des cultes non chrétiens ${ }^{30}$.

En écho à cette littérature, les lettres Indipetae reflètent à la fois une connaissance des classifications des peuples et de leurs conséquences sur les méthodes missionnaires, mais aussi une attirance pour une altérité avec laquelle les Européens espèrent partager quelques caractéristiques. Cela explique sans doute l'importance des demandes pour la Chine et le Japon, ainsi que la propagande de Diego de Torres Bollo, principalement destinée à attirer les missionnaires vers des contrées peuplées par les «barbares» de la troisième catégorie.

\section{Lecteurs et bibliothèques}

La vigueur éditoriale illustre l'engouement du public italien pour les ouvrages portant sur l'Amérique et les terres de mission en général. Les milieux de la marchandise, autant que les humanistes, les juristes ou les ecclésiastiques, sont intéressés par ces écrits, d'où des éditions en langue vernaculaire, accessibles à un large public. Mais il est difficile de mesurer l'impact de ces écrits dans la société en général. On ne rencontre pas des Menocchio tous les jours dans les $\operatorname{archives}^{31}$. C'est pourquoi, la plupart des historiens étudient en priorité les manifestations intellectuelles de cette conscience de l'élargissement du monde. Une histoire sociale de l'impact du Nouveau Monde en Europe reste à écrire.

Les bibliothèques vénitiennes privilégient les grandes synthèses géographiques et présentent une grande variété de livres concernant l'Amérique, parfois plus nombreux que ceux qui traitent de l'Asie ${ }^{32}$. Les motivations des lecteurs seraient diverses: la curiosité pour une littérature qui stimule vers un voyage imaginaire et qui fournit des réponses à des questions théologiques, juridiques, pastorales et morales; une curiosité de collectionneur de livres rares et curieux. Les lecteurs vénitiens chercheraient la confirmation de la supériorité de leur propre civilisation dans la lecture d'œuvres qui présentent le reste du monde sous une forme anecdotique facile d'accès, comme la Cosmographie de Münster, le Theâtre d'Ortelius, les Costumi de Boemus ou l'œuvre de Botero, qui sont les grands succès éditoriaux de l'époque.

29. G. BOTERO aborde la question de la religion dans le monde dans une lettre au cardinal Antonio Carafa, publiée en latin à Paris en 1585 et à Milan en 1586. Ce Discorso de vestigii, et argomenti de la fede catholica reitrovati nell'India da'Portoghesi, e nel Mondo nuovo da' Castigliani, parait en italien à Rome en 1588. Aldo AlbónICO, Il mondo americano di Giovanni Botero, Milan, Bulzoni, 1990, p. 23-34.

30. Cité par A. AlbónICO, Il mondo..., op. cit., p. 199-200. 1980.

31. Carlo GINZBURG, Le fromage et les vers. L'univers d'un meunier du XVI siècle, Paris, Flammarion,

32. Federica Ambrosini, Paesi e mari ignoti. America e colonialismo europeo nella cultura veneziana (secoli XVI-XVII), Venezia, Deputazione di Storia Patria per le Venezie, 1982. 
Si l'on tient compte des traductions des œuvres majeures sur l'Amérique, on trouve, au premier rang des villes éditrices, Venise puis Rome. Certes moins queVenise, Milan reste un important centre éditorial du livre espagnol, avec pas moins de 71 impressions en espagnol et 724 traductions en italien d'œuvres espagnoles entre 1550 et 1600 , et 28 ouvres en espagnol et 277 traductions entre 1601 et $1650^{33}$.

La bibliothèque Ambrosiana, ouverte au public par Federico Borromeo en 1609, permet de mesurer dans le cadre milanais cet intérêt pour la littérature sur le reste du monde. Borromée avait non seulement des ouvrages imprimés mais aussi des œuvres manuscrites. Il rédigea plusieurs traités, inspirés en partie par les écrits de Botero, à ses côtés depuis 1582, puis son conseiller entre 1586 et $1599^{34}$. Il s'intéressait aux objets ayant des vertus médicinales (plantes, pierre bezoar, ambre, baumes divers), aux Indiens, classés du point de vue de la difficulté à les convertir, au démon, à la hierba mate, cultivée dans la région du Tucumán et du Paraguay. Dans les écrits de Borromée, l’Amérique apparaît à travers les Indiens les plus primitifs du continent. Pour le Pérou ou le Mexique il s'inspire de quelques chroniques de la conquête, ou des écrits de Giovanni Botero, se limitant aux questions religieuses et à quelques curiosités, toujours médiatisées par les missionnaires. Aldo Albónico en conclut sans doute rapidement à une crise de la culture à Milan, liée à la crise générale, dont les Milanais ne se relèveraient en ce qui concerne l'Amérique que dans la seconde moitié du $\mathrm{XVII}^{\mathrm{e}}$. Sa critique vis-à-vis de Borromée ne tient compte ni du contexte religieux de ce début du XVII ${ }^{\mathrm{e}}$ siècle, ni du métier de l'archevêque de Milan. Qu'il s'intéresse aux populations d'Amérique ou d'Asie en tant que récents ou futurs chrétiens n'a rien d'étonnant. L'existence de sa bibliothèque, sa volonté de préserver des contacts directs avec des informateurs vivant sur place (comme on le verra plus loin), montrent que Milan participe activement à un intérêt pour les Indes, orientales ou occidentales, qui dépasse la simple curiosité. Celle-ci se retrouve dans le collectionnisme pratiqué par la famille Settala, dont la collection manifeste l'intérêt de l'élite milanaise pour les objets des contrées lointaines et par conséquent l'importance des familles dans l'éducation à la vocation missionnaire des rédacteurs d'Indipetae ${ }^{35}$.

33. Giovanni CARAVAGGI, «La letteratura spagnola in Lombardia», in P. PISSAVINO, G. SIGNOROTTO (éd.), Lombardia borromaica, Lombardia spagnola (1554-1659), Milan, Bulzoni, 1995, vol. I, p. 233-246.

34. A. AlbóniCO, Il cardinal Federico "Americanista», Roma, Bulzoni, 1990.

35. Gino FOGOLARI, «Il Museo Settala. Contributo per la storia della cultura in Milano nel secolo XVII", Archivio Storico Lombardo, 1900, t. II, p. 58-126; Antonio AIMI, «Il Museo Settala: i reperti americani di interesse etnografico", Archivio per l'Antropologia e l'Etnologia, CXIII, 1983, p. 167-186; A. AIMI, V. De Michele, Alessandro MoRAndotTi (éd.), Musaeum Septalianum una collezione scientifica nella Milano del Seicento, Firenze, Giunti Marzocco, 1984 ; numéro thématique de la revue KOS, 24, juillet-août 1986; Aldo De MADDALENA, "Milan the Great». Milano nelle brume del Seicento, Milan, Cariplo, 1989; A. MORANDOTTI, "Milano tra età spagnola e dominio austriaco: componenti sociali e circolazione artistica", in Olivier BONFAIT (éd.), Geografia del collezionismo. Italia e Francia tra il XVI et il XVIII secolo, Rome, École française de Rome, 2001, p. 163-183. 


\section{ÉLITES, FAMILLES ET RÉSEAUX D'INFORMATION}

Sur les 68 rédacteurs des 161 lettres que nous étudions, nous avons des renseignements tirés des catalogues pour 60 d'entre eux. Ils semblent tous assez jeunes au moment d'écrire leur première lettre recensée dans les archives ${ }^{36}$. La jeunesse des scripteurs et le caractère récent de leur entrée dans l'ordre sont confirmés par leur rang: la plupart sont soit novices, soit sortent à peine du noviciat $^{37}$. Ce profil combinant la jeunesse et une entrée récente dans l'ordre confirme l'idée que même si l'éducation donnée au sein de la Compagnie de Jésus (la plupart sont certainement passés par le collège de Brera en tant que jeunes élèves) a joué un grand rôle dans la motivation pour la mission, elle n'est pas la seule origine de ce désir des Indes. Fabrizio Aiazza explique en 1607 que son désir de partir est arrivé en même temps que celui pour la Compagnie. En 1605, Giovanni Giacomo Pasquale, rédacteur infatigable, écrit, dans une de ses 26 lettres, qu'il est entré dans la Compagnie pour aller aux Indes ${ }^{38}$. D'où l'intérêt de mener l'enquête du côté des origines familiales et sociales de ces jeunes gens et de leur inscription éventuelle dans des réseaux relationnels à l'échelle de la monarchie hispanique.

L'installation des jésuites à Milan, dans les années 1560, après presque une décennie de contacts, coïncide avec la constitution du patriciat et la construction de son autonomie vis-à-vis du roi catholique. L'appui des nobles aux jésuites correspondrait à une adhésion à leurs propositions modérées, notamment sur l'équilibre entre vie mondaine et vie chrétienne, alors que la réforme radicale proposée par Charles Borromée rencontrait la résistance de la noblesse milanaise. Même si celle-ci finit par se couler dans ce moule réformateur, le lien noué avec les jésuites se lit dans les congrégations mariales, notamment dans l'église de San Fedele, la première où ils s'installent en $1567^{39}$. Parmi les rédacteurs des lettres Indipetae, on trouve quelques uns des rejetons de ces familles patriciennes.

On a cependant peu de renseignements sur les origines sociales des rédacteurs. Sur les 68 noms repérés, 28 semblent se retrouver dans la section Famiglie des archives de l'Archivio Statale di Milano. Quelques exemples néanmoins dans cet échantillon permettent de conclure à des liens très étroits avec le patriciat de la ville et du Milanais, et peut-être à un réel appui de la part des parents. Parmi les jeunes jésuites qui écrivent dans ces années, trois

36. L'âge va de 16 ans à 67 ans; 14 ont entre 16 et 20 ans ; 32, c'est-à-dire plus de la moitié, ont entre 20 et 30 ans. Parmi les 13 rédacteurs âgés de plus de 30 ans, six ont moins de 15 ans de permanence dans l'ordre, dont un est novice. Sur l'ensemble des rédacteurs pour lesquels on a des informations, 33 appartiennent à l'ordre depuis cinq ans et moins.

37. Onze seulement sont certainement prêtres, dont 3 profès de 4 vœux et un coadjuteur spirituel. Neuf sont coadjuteurs temporels.

38. FG734 f. ; FG733 f.333-333v, et certaines lettres citées par A. GUERRA, "Per un'archeologia», art. cit.

39. Flavio RURALE, I Gesuiti a Milano. Religione e politica nel secondo cinquecento, Rome, Bulzoni, 1992. 
noms de famille figurent dans les grands arbres généalogiques dressés au début du XVIII ${ }^{\mathrm{e}}$ siècle: ceux de la famille des Rho, des Cignardi et des Settala ${ }^{40}$.

Dans sa lettre du 6 janvier 1609, Costanzo Borroni fait allusion, et c'est une chose rare, à la famille d'un des jeunes jésuites qu'il voudrait comme compagnon. Le père de Giovanni Rho, le "comte Alessandro Rho, qui a bien mérité de la Compagnie, sera fort désolé de son départ, mais il y consentira, si votre paternité souhaite le contenter en lui accordant cette grâce»" ${ }^{41}$. Si Giovanni ne partit jamais en mission, mais mourut à Rome en 1662, après avoir été provincial de Milan et de Naples ${ }^{42}$, son frère Giacomo s'embarqua en 1618 et mourut en Chine en 1638, après avoir participé à la rédaction du calendrier impérial avec le Père Adam Schall et écrit de nombreux ouvrages en chinois ${ }^{43}$. Les Rho sont une importante famille de l'oligarchie milanaise, dont les origines remontent aux années 1430. Ils sont comtes de Borghetto et possèdent d'autres fiefs $^{44}$. Ils sont apparentés aux Borromée, par une alliance conclue au début du $\mathrm{XVI}^{\mathrm{e}}$ siècle, mais surtout aux Visconti par la grand-mère paternelle des deux jésuites, Paula Visconti. L'arbre généalogique de Sittoni di Scozia nous apprend que Paulo Rho, frère de Giacomo et Giovanni, était sénateur à Milan. Un Jeronimo Piatti Rho, sans doute un oncle, obtint en 1608 le titre de chevalier de l'ordre de Saint Jacques, preuve de noblesse et signe de considération de la monarchie espagnole ${ }^{45}$.

L'exemple des frères Cignardi, sans doute moins illustre, est représentatif du patriciat milanais. Le recteur de Crémone relate que Gerolamo Cignardi était le fils "de nobles parents milanais", Ferrante Cignardi, trésorier de Sa Majesté Catholique dans l'État de Milan etVirginia Casati, dont le père fut aussi trésorier, et dont le frère, Alfonso Casati, était ambassadeur de Sa majesté Catholique en Suisse ${ }^{46}$. Les Cignardi ne possèdent pas de fiefs ou de seigneuries dans le Milanais, cependant la famille semble ancienne, l'arbre généalogique de Sittoni di Scozia remontant jusqu'au XIV ${ }^{\mathrm{e}}$ siècle. Ses membres ont noué des liens étroits avec la monarchie espagnole par un fort ancrage dans l'administration financière et diplomatique, dont le rôle revêt une importance primordiale à cette époque troublée de la politique internationale. Les Casati eurent ainsi quasiment le monopole, tout au long du siècle, des relations

40. Giovanni SITTONI DI SCOZIA, Theatrum Genealogicum familiarum illustrium nobilium et civium inclytae urbis Mediolani, 1705. Manuscrits conservés à l'Archivio di Stato de Milan.

41. FG734 f. 246 rv.

42. C. SoMmervogeL, Bibliothèque..., op. cit., vol. VI, col. 1711.

43. C. Sommervogel, Bibliothèque..., op. cit., vol.VI, col. 1709. P. G. Coole, «Nuovi documenti sui Gesuiti in Cina", La Clessidra, n 48, s.d., p. 58-61.

44. Le terme italien est feudo. Enrico CASANOva, Dizionario feudale delle provincie componenti l'antico stato di Milano all'epoca della cessazione del sistema feudale (1796). Ducato di Milano, Principato di Pavia di aqua del Po, Contado di Como, Contado di Cremona, Contado di Lodi, Milan, Biblioteca ambrosiana, 1930, p. $12,19,42,62,109$.

45. Madrid, Archivo Histórico Nacional: 12.2.1. OM-CABALLEROS-SANTIAGO, EXP. 6447. 46. ARSI, VITAE 84 f. 56. 
diplomatiques avec la Suisse toute proche et qui occupe une place stratégique de premier ordre en raison du statut de la Valteline ${ }^{47}$.

On trouve également parmi les rédacteurs un certain Claudio Francesco Settale, l'aîné des dix-huit enfants de Ludovico (1552-1633), un médecin milanais célèbre, ami des Borromée. Son frère, Manfredo Settala (1600-1680), prolongea le goût pour le collectionnisme de son père par la création d'un véritable musée visité par toute la bonne société milanaise du XVII ${ }^{\mathrm{e}}$ siècle ${ }^{48}$. Armes, tissus, objets d'usage quotidien du Brésil et du Chili composaient la célèbre collection de trouvailles ethnographiques américaines des Settala, approvisionnés entre autres par le jésuite Alonso de Ovalle ${ }^{49}$. Pourtant Claudio Francesco Settala explique en 1605 qu'il ne connaît l'existence des missions d'Ethiopie et de Chine que depuis son entrée dans la Compagnie ${ }^{50}$. La collection Settala passa dans la bibliothèque Ambrosiana au XVIII ${ }^{\mathrm{e}}$ siècle et une partie est toujours visible aujourd'hui.

Appartenant à un milieu où circulaient les livres, les objets exotiques, mais aussi les hommes, ces jeunes jésuites étaient amenés à fréquenter les autorités espagnoles et d'autres cercles intéressés par le vaste monde, comme ceux des collectionneurs, mais aussi des militaires ou des administrateurs, dont la carrière a pu se dérouler des deux côtés de l'Atlantique et même aux Philippines. La mission lointaine, à laquelle les compagnons d'Ignace avaient un accès privilégié, a pu séduire ces jeunes gens, mais aussi leurs familles, dans la perspective d'accéder à l'horizon impérial de la Monarchie catholique espagnole et de maintenir Milan au rang de capitale. L'horizon missionnaire avait un sens social et religieux pour cette élite actrice de la réforme catholique, comme le montre par exemple l'amitié entre un missionnaire jésuite et l'archevêque Federico Borromeo, qui révèle les liens directs entre la société milanaise dévote et les missionnaires.

\section{ÉGLISE, SOCIÉTÉ DÉVOTE ET MISSIONS LOINTAINES}

La correspondance entre Federico Borromeo et Diego de Torres Bollo, fondateur et supérieur de la province du Paraguay, se compose de quatorze lettres, sur une période de plus de 20 ans, entre 1603 et 1625 (elle est conservée à la Biblioteca Ambrosiana). Procureur de la province jésuite du Pérou, il rencontre F. Borromeo lors de ses deux passages à Milan sur la route de Rome, à l'automne 1602 puis au printemps 1603. À Milan, il loge chez Pedro Enriquez de Acevedo, comte de Fuentes, gouverneur espagnol du Milanais de 1601 à 1610, en bons termes avec l'archevêque ${ }^{51}$. Lors de ces séjours, Torres rencontre certains des

47. G. SignorotTo, Milano Spagnola..., op. cit. p. 40. Giovanni MUTO, «Il governo della hacienda nella Lombardia spagnola ", in P. PISSAVINO, G. SignOROTTO (éd.), Lombardia ... op. cit., vol. I, p. 265-302.

48. G. FOGOLARI, «Il Museo Settala», art. cit.

49. A. Albònico, Il cardinal Federico "Americanista", Roma, Bulzoni, 1990.

50. FG733 f. 342-342v.

51. Storia di Milano, t. X, L'età della riforma cattolica (1559-1630), Milan, Fondazione Treccani degli Alfieri per la storia di Milano, 1957, p. 325. F. RURALE, "Questioni di politica ecclesiastica tra Roma e Milano", Studia Borromaica, 18, 2004, p. 63-95. 
jésuites de la province de Milan qu'il emmène dans l'expédition qu'il organise vers le Pérou et la nouvelle province du Paraguay en $1604^{52}$. La longue relation d'amitié entre le jésuite espagnol et l'archevêque revêt des dimensions multiples, à l'échelle de la monarchie espagnole, dans le cadre de la Rome pontificale, mais aussi dans les milieux dévots milanais.

Cette relation s'inscrit dans la logique des réseaux ecclésiastiques et politiques de l'archevêque. Il a passé de nombreuses années à Rome, où vit sa cousine, Ortensia Borromeo, sœur de Carlo et épouse de Marco Sittico Altemps, à qui il doit certainement sa pourpre cardinalice. C'est dans la célèbre villa Altemps qu'il aurait rencontré Filippo Neri, fondateur de l'Oratoire de Rome, mort en 1595 et son ami Cesare Baronio, candidat malheureux à la papauté en 1605, après le décès de ClémentVIII. Dans la correspondance, Torres Bollo fait plusieurs fois allusion à Baronio, dont il espère l'élection, qu'il estime bénéfique aux missions du Paraguay ${ }^{53}$. À Rome, Torres Bollo fut le procureur de l'archevêque de Lima auprès du pape dans le cadre de la visite ad limina et joua certainement un rôle dans les premiers projets de création d'un collège pour la formation des missionnaires, à l'instar de Martin de Funes ${ }^{54}$. En Espagne, Torres demanda l'appui du roi pour obtenir la canonisation de Charles Borromée (effectuée en 1610).

Le séjour de Torres Bollo à Milan lui permit, par l'intermédiaire de l'archevêque, mais aussi sans doute de Martin de Funes, de rencontrer les milieux dévots milanais. Il affirme correspondre avec les prieurs et les députés d'une congrégation milanaise de San Dalmazio, demandant à Borromée de les informer de ses lettres ${ }^{55}$. En 1605, il charge le procureur de sa province en Europe de visiter la congrégation de sa part, signe de la volonté de maintenir un lien étroit avec le patriciat milanais. Vingt ans plus tard, il recommande à Borromée un procureur du Pérou à Rome et lui demande de lui permettre de «dire la messe sur l'autel de Saint Charles, de lui montrer la fervente congrégation de la doctrine et son insigne librairie». En 1608, il demandait aussi à Borromée de recommander à Dieu «la conversion de cette gentilité» et d'inciter les écoles de la doctrine chrétienne de la ville de Milan à faire de même ${ }^{56}$.

52. L'infirmier qui l'a soigné en Italie, Agostino Salumbrini, deux jeunes étudiants, dont on a retrouvé la lettre Indipeta et Martin de Funes, un jésuite espagnol important par la suite dans la province de Nouvelle Grenade. Martin Morales, (éd.), A mis manos han llegado. Cartas de los PP. Generales a las antigua provincia del Paraguay (1608-1639), Madrid-Rome, IHSI, 2005, p. 151. Giuseppe PIRAS, Martin de Funes, S. I. (1560-1611) e gli inizi delle Riduzioni dei gesuiti nel Paraguay, Rome, Edizioni di storia e letteratura, 1998.

53. Lima, le 8 décembre 1604, Biblioteca Ambrosiana Manoscritti (désormais BAM) 251, 41.

54. G. PIRAS, La Congregazione e il Collegio di Propaganda Fide di F. B.Vives, G. Leonardi e M. de Funes, Rome, Universitá gregoriana editrice, 1976.

55. Lima, 8 décembre 1604, BAM, 251, 41. Une deuxième allusion dans la lettre du 23 juillet 1606, Santa Fe de Nuevo Reyno: BAM, 251, 237 et 240.

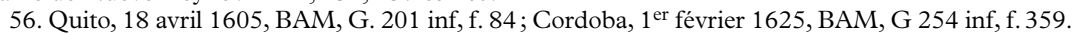
Santiago de Chile, 25 mars 1608, BAM, 199, 294. Quelques lettres ont été publiées par Giovanni GALBIATI, "Notizie sulle antichità sudamericane del Museo Settala all'Ambrosiana", Atti del XXII Congresso degli americanisti, Rome, Istituto Cristoforo Colombo, 1928, vol. I, p. 512-520. 
La congrégation de San Dalmazio regroupe des oblats du Saint Sépulcre, c'est-à-dire des laïcs ou semi-religieux qui s'occupent d'une école de la doctrine chrétienne depuis 1577, avec l'approbation de Charles Borromée ${ }^{57}$. La seule église de San Dalmazio (à côté de la Scala) de Milan fut fermée au $\mathrm{XVIII}^{\mathrm{e}}$ siècle et détruite en 1786. Chère à Federico Borromeo, l'église aurait conservé la caisse où Charles avait reposé juste après son décès. Elle était considérée comme la "métropole" des écoles de la doctrine chrétienne, œuvre de Charles continuée par Federico ${ }^{58}$. Une gravure de 1630 représente les élèves de cette école dans la petite église de San Dalmazio, au plafond en bois, avec Carlo et Federico en habit de cardinaux, priant devant un Christ en croix ${ }^{59}$. Selon un des premiers biographes de FedericoBorromée, l'archevêque y emmenait toutes les personnes de qualité qui passaient par Milan. Lors de son passage par Milan en 1599 pour rejoindre son mari, Philippe III, Marguerite d'Autriche se serait inscrite dans ces écoles de doctrine chrétienne. L'archevêque Borromée figure avec un don de 300 écus dans les documents comptables de la congrégation ${ }^{60}$. En 1630, l'enseignement dispensé à San Dalmazio concernerait 34749 personnes. Torres la fréquenta lors de son passage dans la ville, peut-être par l'intermédiaire de Martin de Funes, ancien précepteur de la princesse Marguerite à Graz et son professeur d'espagnol lors de son séjour milanais. Dans le compte rendu d'une réunion des membres de la congrégation de la Doctrine Chrétienne de San Dalmazio, du 20 janvier 1628 conservée à l'Archivio di Stato, on trouve parmi les membres, le nom de famille d'un des rédacteurs d'Indipeta, Andrea Caponago.

Les écoles de la doctrine chrétienne, contrôlées à Milan par l'archevêché, constituent une des activités menées par les laïcs dans le cadre des confréries tournées vers la charité, l'édification personnelle et l'assistance aux pauvres et aux malades. Ces activités s'ajoutent à celles des confréries de pénitence héritées du Moyen Âge qui sont renouvelées au moment de la réforme catholique ${ }^{61}$. Le rappel que fait Torres Bollo de l'activité d'enseignement de la doctrine chrétienne menée par des laïcs dans le cadre urbain constitue un exemple de circulation des méthodes d'évangélisation. En 1612, Torres Bollo demande encore une fois à Borromée de saluer "le préfet et les officiers de la doctrine», qu'il

57. Carlo TORRE, Il rittratto di Milano, Milan, 1714 (1674 ?), p. 233; Archivio di Stato di Milano (désormais ASM), Fondo Religione, 545, S. Dalmazio Congregazione della Dottrina Cristiana. Circondario 1579-1785.

58. Dizionario della Chiesa ambrosiana, vol. 2, Milan, Egidio Boniardi, 1988, p. 988; C. TORRE, Il rittratto..., op. cit., p. 233. Francesco Rivola, Vita di Federico Borromeo, Milan, Dionisio Gariboldi, 1656, p. $260-271$.

59. BAM X 308 inf, f. 62. La gravure accompagne un texte à remplir par le professeur de doctrine chrétienne attestant que l'élève a bien fréquenté l'école. C. TORRE explique qu'on y trouvait une fresque représentant les deux cousins Borromée, Il rittratto..., op. cit., p. 233.

60. F. RIVOLA, Vita..., op. cit., p. 260-271. ASM, Fondo Religione, 545, S. Dalmazio, Congregazione della Dottrina Cristiana. Circondario 1579-1785.

61. Storia di Milano..., op. cit., p. 175. David GARRIOCH, «La persistance des confréries milanaises au XVIII ${ }^{\mathrm{e}}$ siècle", Revue d'histoire moderne $\mathcal{E}$ contemporaine, 52-4, octobre-décembre 2005, p. 50-71. 
remercie de se souvenir de lui et dont il «répète de nombreuses fois ce qu'il les a vus faire" à Milan ${ }^{62}$.

Torres Bollo exerce d'abord comme supérieur de la province de Nouvelle Grenade, ce qui explique qu'il écrive aussi depuis Santa Fe de Bogotá. Il part ensuite fonder la province du Paraguay où il termine sa vie. Les lettres qu'il envoie à l'archevêque évoquent régulièrement l'état de ces provinces et les conditions de travail des missionnaires. Comme dans l'ouvrage qu'il publie en 1603, il parle en priorité des Indiens non baptisés qui restent à convertir aux marges de l'empire espagnol, sans aucun mot sur les missions parmi les Indiens convertis. Il compte assouvir la curiosité des Européens et susciter leur générosité, en échange de la bienveillance de Dieu. En 1604 il insiste sur les «innombrables infidèles" qu'on trouve dans la future province du Paraguay, où les jésuites sont déjà installés depuis les années 1580 pour «découvrir de nouveaux trésors d'âmes et peuples de gentils, parce que, comme beaucoup le disent, c'est ici que se trouve la porte pour découvrir un nouveau monde, inconnu jusqu'à maintenant ${ }^{63}$. Annonçant son prochain départ, il évoque la présence au Paraguay des «nations les plus barbares qu'on trouve dans ces terres, qui sont les anthropophages qui mangent les hommes, aux coutumes particulièrement féroces et jugés incapables du saint évangile par beaucoup». Dans le Royaume du Chili, sous la juridiction du Paraguay "vivent d'innombrables Gentils, et les plus belliqueux que nous connaissions aujourd'hui dans toutes les Indes " ${ }^{64}$. Plus tard il informe Borromée des progrès des réductions et se plaint surtout de l'incurie des Espagnols, occupés dans la région à faire le commerce de la nerba mate au détriment des Indiens, souhaitant que l'archevêque intervienne auprès de l'évêque du Paraguay en faveur des missions ${ }^{65}$.

Aux informations sur les populations s'ajoutent les objets destinés à remplir les collections de Borromée. En 1605, Torres Bollo lui annonce l'envoi d'un livre de Chine, d'une pierre miraculeuse et d'un baume. En mai 1607, il lui envoie une grammaire trilingue et des pierres «bezoares", visibles aujourd'hui dans la collection du musée de la bibliothèque ambrosienne ${ }^{66}$.

Très régulièrement, il évoque la pauvreté matérielle des provinces jésuites en cours de création et le sentiment d'abandon que ressentent les missionnaires. C'est le sens de son allusion à l'éventuelle élection de Baronio comme pape, avec l'espoir qu'il favorise le ministère des missions. En 1605, il déplore le manque de missionnaires et annonce l'envoi de son propre compagnon, le Père Juan Antonio, pour demander secours à Rome. Il espère que l'archevêque, nommé patron de la province du Paraguay, l'appuiera dans ses démarches en Italie ${ }^{67}$.

62. Chili, 10 octobre 1612, BAM, G. 253 inf, f. 102.

63. Lima, 8 décembre 1604, BAM, 251, 41.

64. Lima, 20 mai 1607, BAM, 196, 53.

65. Tucumán, 11 juillet 1623, BAM, G 237 inf, f. 93.

66. Quito, 18 avril 1605, BAM, G. 201 inf, f. 84. Lima, 20 mai 1607, BAM, 196, 53.

67. Quito, 18 avril 1605, BAM, G. 201 inf, f. 84. 
Il évoque la joie des jésuites de Lima à la lecture d'une lettre de Borromée adressée à l'un d'eux qu'ils ont l'intention de traduire en espagnol pour qu'elle circule $^{68}$. En 1605, il demande à Borromée une aumône pour habiller et nourrir ses missionnaires du Paraguay. Puis en 1608, il sollicite l'envoi de nombreuses reliques avec un procureur de la province et demande aussi à Borromée de faciliter le départ de jésuites italiens pour la province du Paraguay ${ }^{69}$.

Le cardinal sert de lien avec le collège de Brera à Milan, en lui transmettant un récit des missions de Santa Fe, "pour les animer à partir les aider». La traduction en italien de la lettre fut légèrement expurgée, avec la suppression des difficultés rencontrées par Torres Bollo lors de l'expédition qu'il organisa en $1604^{70}$. Faut-il croire que Torres Bollo se méfie des supérieurs de la province milanaise et préfère utiliser d'autres voies pour diffuser sa propagande pour la mission?

Les demandes d'aumônes sont apparemment suivies d'effet puisqu'en mai 1612 le procureur de Séville pour la Compagnie de Jésus en Amérique remercie dans un courrier l'archevêque Borromée pour la caisse de calices reçue via Gênes, qu'il s'apprête à envoyer à Torres Bollo. Celui-ci accuse réception en mars 1617 depuis Cordoba des calices et de la relique de Charles, à peine arrivés à Buenos Aires. En 1621, des rosaires attendent dans le port sudaméricain, alors qu'en 1623, Torres Bollo annonce à Borromée que les reliques de Charles sont au collège jésuite d'Asunción, au Paraguay ${ }^{71}$.

À travers Borromée, c'est à la société milanaise que s'adressent ces demandes d'aumône et de prières, notamment par l'intermédiaire des écoles de la doctrine chrétienne. La sollicitation concerne l'ensemble du diocèse lorsqu'en 1608 Torres Bollo explique que plus de 400 femmes espagnoles issues de la noblesse ont été faites prisonnières par les Indiens sauvages du Chili et qu'elles ont sans doute eu des enfants qui n'ont pas été baptisés. Il «espère obtenir une bonne aumône que Son Illustrissime Seigneurie commandera de faire dans ce très riche diocèse de Milan" et fait appel à sa "noblesse", promettant à ses «sujets" la gloire dans l'autre vie. L'accent mis sur la "noblesse» est tout à fait volontaire, puisque le terme "noble» est écrit quatre fois dans la lettre ${ }^{72}$. Torres Bollo connaît les références de la société européenne et sait quelles cordes sensibles il vaut mieux toucher. En décrivant la captivité de ces femmes et de leurs enfants, il explique que «dans les mains de ces barbares ils sont si maltraités, que l'esclavage parmi les Turcs n'est pas aussi cruel et inhumain que celui-ci, parce qu'ils se servent de ces pauvres captifs comme instruments pour les tâches les plus viles et les plus fatigantes". À la noblesse de ces femmes

68. Lima, 8 décembre 1604, BAM, 251, 4.

69. Lima, 20 mai 1607, BAM, 196, 53. Santiago de Chile, 25 mars 1608 BAM, 199, 294. Chili, 10 octobre 1612, BAM, G. 253 inf, f. 102.

70. Santa Fe de Nuevo Reyno, 23 juillet 1606, BAM, G 251, 237 et 240.

71. Séville, 20 mai 1612, BAM, G. 211 bis inf, f. 256. Cordoba, 16 mars 1617, BAM, 253, 204. Cordoba, 19 mars 1621, BAM, 254, 158. Tucumán, 11 juillet 1623, BAM, G 237 inf, f. 93.

72. Santiago de Chile, 2 avril 1608, BAM, 199, 317. 
s'oppose le travail dégradant auquel elles sont réduites, ce qui est tout à fait choquant pour une société attachée au statut, à la bienséance et à la civilité.

La description qu'il fait des Indiens constitue également pour le missionnaire un moyen de s'inscrire dans une culture partagée par les hommes de son temps. Il met systématiquement l'accent sur la barbarie des Indiens des frontières de la chrétienté, qui correspondent à la troisième catégorie décrite par José de Acosta, reprise par Giovanni Botero, celle des Indiens sans foi ni loi. À l'instar de la comparaison avec les Turcs, l'emploi comme synonymes des termes «infidèle» ou "païen ", alors qu'une distinction très claire existe entre ces deux qualificatifs, n'est pas innocente. Le jésuite simplifie le vocabulaire pour rappeler à l'archevêque et à ses ouailles leurs propres angoisses vis-à-vis des Ottomans, qui sont alors aux portes de l'empire des Habsbourg. La même ambiguité sur les Indiens des marges de l'empire espagnol qui restent à convertir est perceptible dans l'ouvrage publié par le missionnaire en 1603. Ainsi, au moment où Torres Bollo sensibilise le patriciat milanais à la conversion des Indiens, par l'intermédiaire de l'archevêque Borromée, il peut être lu sur ce même thème en latin, français, italien, et même en polonais dès 1603-1604.

On ne sait pas si les fidèles du Milanais ont envoyé de l'argent à Torres Bollo pour secourir les nobles femmes captives des Indiens Araucans. Il est possible qu'ils en aient eu connaissance par Borromée ou par d'autres vecteurs. Lorsqu'on envisage les moyens par lesquels il était possible aux missionnaires de s'adresser à un public européen dans son quotidien et dans le cadre de sa pratique religieuse, il est difficile de ne pas penser à la messe, à la prédication, aux processions et à la débauche d'images produites par la culture baroque de la réforme catholique. Les archives conservent peut-être un sermon de Borromée faisant allusion à l'Amérique ou à la conversion des gentils. Le XVII ${ }^{\mathrm{e}}$ siècle se caractérise par une utilisation accrue des exempla, semblables à ceux convoqués par les mendiants à l'époque médiévale et puisant dans les mêmes modèles. En Nouvelle Espagne ces petites histoires utilisées par les prédicateurs pour appuyer l'argumentation tendaient à construire le modèle d'un "pécheur universel» en utilisant des récits glanés dans le monde entier, adaptés aux réalités mexicaines. Très nombreuses dans les lettres annuelles qui circulent en Europe, celles qui figurent dans la correspondance de Torres Bollo présentent des Indiens vertueux refusant de retomber dans le paganisme. Danièle Dehouve a montré que les exempla américains sont ensuite réutilisés dans la littérature européenne, notamment espagnole ${ }^{73}$. La prédication à Milan a pu puiser à ce type de source venant d'Asie ou d'Amérique, faisant ainsi connaître à la société urbaine européenne des récits sur les populations bigarrées des territoires de conversion ${ }^{74}$.

73. Danièle DeHouve, L'évangélisation des Aztèques ou le pécheur universel, Paris, Maisonneuve \& Larose, 2004

74. À la prédication, il faut aussi ajouter les processions et les célébrations à l'occasion de béatifications ou de canonisations, comme ce fut le cas pour les fêtes dédiées à Ignace et à François Xavier en 1622. Le théâtre, mais aussi la statuaire, comme celle consacrée à l'idolâtrie à Rome, constituent d'autres pistes à cet égard. 
Chez les jeunes jésuites milanais rédacteurs de lettres Indipetae, la curiosité est susceptible de se transformer en action. La mission lointaine suscite des controverses, des discussions contradictoires, une grande effervescence et parfois même la fabrication de véritables mythes.

\section{LE MYTHE DU DÉPART : ENTRE LIENS AMICAUX ET IMAGINAIRE COLLECTIF}

La relative rareté de l'élection des jésuites italiens pour les expéditions de missionnaires envoyées dans les possessions portugaises et espagnoles au début du XVII ${ }^{\mathrm{e}}$ siècle explique sans doute en partie l'ardeur des rédacteurs d'Indipetae de notre corpus, mais aussi la construction d'une véritable mythologie du départ en mission, qui révèle l'importance de cet horizon lointain dans l'imaginaire collectif de cette fraction de la jeunesse.

Une des principales difficultés auxquelles doivent faire face les candidats est l'attitude des supérieurs locaux: quelques-uns opposent une résistance active à l'écriture de la lettre et à l'éventuel départ, alors que d'autres, souvent candidats malheureux eux-mêmes, encouragent les jeunes gens à se manifester au général. Lorsque les supérieurs des provinces sont cités, c'est souvent pour évoquer leur résistance à laisser partir des recrues de qualité, utiles pour des tâches d'enseignement. En 1609, Carlo Torniello explique qu'il demande la mission depuis sept ans. Bien qu'il ait été choisi trois ans auparavant, le provincial l'avait retenu à Crémone pour le cours de rhétorique. Il demande une réponse directe au général et soupçonne les supérieurs de vouloir l'empêcher de partir sous prétexte que "les Indes sont ici». En 1609, Alfonso Cignardi évoque l'opposition de son provincial à son éventuel départ pour les Indes. Giovanni Rho dit la même chose, soulignant les bonnes dispositions du général ${ }^{75}$.

En revanche, Fignoli et Cignardi citent leur maître des novices, Tomaso Ceronio, qui les encourage et leur a peut-être fourni des informations précises en décembre 1606. Lui-même rédigea deux lettres Indipetae en 1603. En 1606 et 1611 , il est maître des novices à Arona, où il a certainement continué à encourager les jeunes jésuites au départ en mission ${ }^{76}$. Le 6 janvier 1609, Fabrizio Aiazza est soutenu par son professeur de philosophie, Costanzo Borroni, qui écrit le même jour, confirmant au général la mauvaise volonté des supérieurs. Quand il avait été candidat neuf ans auparavant, son supérieur lui avait caché la réponse positive du général pour partir avec le procureur d'Inde, le P. Laertio. Il désire pour lui-même une réponse directe ${ }^{77}$. Une série d'ordres donnés avant 1619 à la province de Milan, par le général Vitelleschi, confirme cette tension entre les candidats au départ, les supérieurs locaux et le général. On y demande

\footnotetext{
75. FG734 f. 171rv. FG734 f. 311-311v. FG734 f. 284-284v.

76. FG733 f. 451-451v. ARSI, Med. 47, catalogue de Milan 1606, f. 223, p. 24, n 1 .

77. FG734 f. 247-247v. FG734 f. 246-246v.
} 
que «personne ne dissuade les nôtres d'aller en Inde, ou n'en parle autrement qu'il ne convient ${ }^{78}$.

Dans la même lettre du 6 janvier 1609, Borroni oppose l'adhésion des parents à l'attitude des supérieurs. Son allusion à Alessandro Rho, le père de Giovanni et de Giacomo, nous renseigne sur l'importance de l'avis des parents dans le destin des jeunes nobles milanais, même après leur entrée en religion. Elle soulève la question de l'autorité paternelle dans les choix d'avenir des jeunes nobles européens à qui s'offrait l'alternative entre le mariage et le célibat sous plusieurs formes, dont l'entrée en religion. Renata Ago a montré que les choix des parents, liés à des impératifs lignagers, tenaient néanmoins compte du désir et de la vocation des enfants ${ }^{79}$. Passée la porte du couvent ou, comme ici, du collège, les parents restent présents. Souvent opposés au départ de leurs enfants pour la mission lointaine, comme le montre Charlotte de Castelnau pour le Portugal ${ }^{80}$, ils semblent également soucieux du désir de leur enfant. Alessandro Rho se décharge de la décision sur le général de la Compagnie de Jésus, à qui il a délégué son rôle de père lorsqu'il y a laissé entrer deux de ses fils. Le lien familial demeure néanmoins étroit, comme le montre la correspondance de Giacomo Rho avec son père et d'autres membres de sa famille après son départ pour l'Inde en 1618, publiée en 1620 à Milan et traduite en allemand $^{81}$. Selon Renata Ago, ce souci pour la liberté de choix des jeunes gens s'appuie, particulièrement en Italie, sur l'importance du libre-arbitre dans la théologie post-tridentine. L'existence des lettres Indipetae le confirme, dont l'écriture est en elle-même à la fois un choix résultant d'un examen de conscience approfondi des candidats et une preuve matérielle pouvant être produite en cas de réclamation familiale.

Une fois ces difficultés surmontées, le départ des candidats italiens à la mission dépend du rythme des expéditions, du choix des supérieurs à Rome, mais aussi de la relative ouverture des royaumes ibériques à l'arrivée d'Italiens. Si on examine les expéditions en Inde, c'est-à-dire vers Goa, porte de l'ExtrêmeOrient pour les missionnaires contrôlée par les Portugais, elles sont quasiment annuelles entre 1597 et 1611 (sauf trois années sans départs, en 1598, 1606 et $1610)^{82}$. Sur un total de 225 missionnaires, la plupart du temps portugais, on trouve environ un tiers d'Italiens (78), avec ponctuellement une proportion plus

78. ARSI, Med 76 II, f. 449-450: «Ordini di NP Generale datti alla Provincia di Milano in diverse lettere al Prov. le Bernardino Rossignolo, e da lui intimato a tutti i superiori".

79. Renata AGO, "La liberté de choix des jeunes nobles au XVII" siècle», in Giovanni LEVI, JeanClaude SCHMiTT (éd.), Histoire des jeunes en Occident, de l'Antiquité à l'époque moderne, Paris, Seuil, 1996, p. 331-377.

80. C. DE CASTELnAU-L'Estoile, "Élection et vocation: Le choix de la mission dans la province jésuite du Portugal à la fin du XVI ${ }^{\mathrm{e}}$ siècle» in B. VINCENT, P.-A. FABRE (éd.), Notre lieu..., op. cit., p. 21-43.

81. Lettere del P. Giacomo Ro Della Compagnia di Giesú doppo la sua partenza di Lisbona per la Cina, che fù alli 6 d'Aprile 1618. Scritte al Signor Alessandro Ro I. C. suo Padre in mezo al Océano..., In Milano, Apresso Gio. Battista Bidelli, X.D.XX. C. Sommervogel, Bibliothèque..., op. cit., t. 6, col. 1709-1710. Bidelli est l'éditeur de nombreuses œuvres de jésuites milanais.

82. ARSI, Goa 28 Elencus missorum in Indiam, 20 folios. 
importante, comme en 1602, lorsque le Père Laertio partit avec 60 missionnaires, dont 30 Italiens. Entre 1597 et 1611, un seul Milanais figurant dans le corpus des rédacteurs étudié ici, Ludovico Mariana, est choisi, en 1607.

Le passage en Amérique est susceptible d'emmener au Brésil (via le Portugal), en Nouvelle Espagne, étape sur le chemin des Philippines, ou au Pérou, en Nouvelle Grenade, au Paraguay et au Chili. L'expédition la plus importante pour le Pérou fut réunie par le père Diego de Torres Bollo en 1603, avec 40 missionnaires dont 13 Italiens. Dans la province du Pérou les Italiens avaient une réputation de missionnaires zélés qui apprenaient facilement les langues indiennes ${ }^{83}$. En principe, seuls les sujets de la Couronne étaient autorisés à passer en Amérique par la Casa de la Contratación, Philippe III ayant précisé entre 1598 et 1602 l'interdiction faite aux Italiens ${ }^{84}$. Contournant cette disposition, Torres Bollo fit apparaître leurs noms dans les listes officielles sous une forme hispanisée. La jeunesse des jésuites de cette expédition n'est pas un obstacle, puisque nombreux sont les novices choisis, signe de la réticence des provinces d'Europe à se séparer d'individus déjà formés mais aussi de la confiance que l'institution accorde aux établissements fondés dans les provinces lointaines, capables de fournir une formation intellectuelle et religieuse de qualité 85 .

Ces jeunes novices, ou déjà étudiants, sont une population spécifique, qui vit en groupe au sein de structures collectives où ils partagent des amitiés souvent depuis l'enfance. Malgré la nature individuelle du choix que révèle l'écriture de la lettre Indipeta, c'est collectivement qu'ils affrontent la rareté de l'élection et les obstacles rencontrés de la part de l'autorité familiale ou religieuse. En dehors des lettres Indipetae, d'autres textes évoquant le départ en mission invitent à s'interroger sur la construction, encouragée par l'institution, de véritables mythes autour de la vocation missionnaire et de sa réalisation.

Parmi les scripteurs de ce corpus milanais, quelques cas sont à mettre en valeur, soit parce qu'ils ont écrit de nombreuses lettres, soit parce qu'ils rédigent des lettres collectives. Giovanni Battista Bonelli, Alfonso Cignardi et Gerolamo Fegnoli écrivent ensemble en 1606 une courte lettre, alors qu'ils n'ont pas encore terminé le noviciat. En 1608, Bonelli et Fignoli font à nouveau équipe et rappellent au général leur désir, arguant du fait qu'ils ont gagné en maturité et qu'on ne peut plus les soupçonner d'"esprit de noviciat", puisqu'ils ont presque fini le cours de logique. Le 6 janvier 1609, Costanzo Borroni confirme l'existence de ce groupe en faisant un éloge détaillé de Fabrizio Aiazza, Carlo Torniello, Giovanni Rho et Alfonso Cignardi qu'il désire avoir comme compagnons dans le cas où il partirait ${ }^{86}$.

83. A. MALDAVSKY, "L'administration....", art. cit.

84. Lázaro AsPuRZ, La aportación extranjera a las misiones españolas del Patronato Regio, Madrid, Consejo de la Hispanidad, 1946.

85. A. MALDAVSKY, "L'administration..." , art. cit.

86. FG733 f. 451-451v. FG734 f. 174rv. FG734 f. 246-246v. 
Cette réelle effervescence et cet esprit missionnaire dont on soupçonne l'existence dans les collèges et noviciats sont très bien illustrés par une histoire d'apparition qui concerne Gerolamo Cignardi, le frère d'Alfonso, et Giovanni Battista Bonelli. Le 31 janvier 1608 meurt à Crémone Gerolamo Cignardi, terrassé à vingt ans par une maladie respiratoire, un an après avoir écrit son unique Indipeta. Très rapidement, sa réputation de saint suscite des récits qui racontent sa courte vie et comment il est apparu à l'occasion de sa mort à son meilleur ami, Giovanni Battista Bonelli. Celui-ci avait rédigé une lettre avec Alfonso, le frère de Gerolamo et d'autres. Il partit en Orient en 1620, où il mourut en 1638, trente ans après son ami Gerolamo.

Quatre récits restituent l'apparition qui donna à Gerolamo une réputation de saint. L'un d'eux (1) fut sans doute rédigé par le recteur de Crémone au moment du décès ${ }^{87}$. Dionisio Sivori, qui dit lui-même avoir été un «de ses compagnons dans le siècle, au noviciat et au collège de Côme, en classe de rhétorique" rédigea un autre récit (2) "della vita della santa memoria del fratello Geronimo Cignardi " 88 et une lettre sur Giovanni Battista Bonnelli (3), écrite de Crémone le 3 mai 1668 au F. Giovanni Battista Ambrosino, pour servir à l'histoire de l'Ordre en Italie que préparait alors le P. Bartoli ${ }^{89}$. Elle inclut une narration de la grâce obtenue par l'intermédiaire de la relique d'un index entier de Cignardi, avec la chair et la peau, qui aurait eu lieu à Posnan, en Pologne, bien des années plus tard ${ }^{90}$. Les archives conservent également une «Narratione dell'apparitione fatta dal Fllo Gerolamo Cignardi al P. Gio. Battista Bonnelli scritta dal P. Alessandro de Rodes nella relatione del Tonchino capo. XLVI» (4), copie manuscrite du chapitre correspondant, publié en italien en $1650^{91}$. Alexandre de Rhodes raconte la mort du Père Bonelli en 1638, alors qu'il traversait les montagnes entre le Tonkin et le Laos, en tant que visiteur de la mission du Tonkin, après de nombreuses années passées à Macao ${ }^{92}$.

Trois de ces récits $(1,2$ et 4$)$ racontent grosso modo la même histoire. Très bons amis, Bonelli et Cignardi s'étaient promis de se manifester l'un à l'autre au moment de leur mort. Alors que Bonelli était à Milan, au collège de Brera, Cignardi lui serait apparu à l'aube pour lui signaler sa propre mort. Bonelli aurait demandé à son ami ce qu'il adviendrait de lui-même et Cignardi lui aurait dit qu'il avait encore un long chemin devant lui. Pour Alexandre de Rhodes, il s'agit d'une prémonition de son avenir de missionnaire, puisqu'ils avaient tous deux écrit une lettre Indipeta.

Un autre récit (3), restitué par Sivori en 1668, soixante ans après la mort de Cignardi, 30 ans après celle de Bonelli, et 18 ans après la publication de

87. ARSI, VITAE 84, f. 55-78v : «Vita di Gerolamo Cignardi della C. di Gesu».

88. ARSI, VITAE 85, f. 32-33.

89. ARSI, VITAE 168 , f. 51-52

90. ARSI, VITAE 168 f. $2-3$.

91. ARSI, VITAE 86, f. 212-213.

92. Alexandre DE RHODES, Relazione De'Felici successi della Santa Fede predicata da' padri della Compagnia di Giesu nel regno di Tunchino, Rome, Giuseppe Luna, 1650. 
l'œuvre d'Alexandre de Rhodes en italien, diffère très largement des versions précédentes et inscrit cet événement dans le contexte de l'effervescence missionnaire dans les collèges. Il raconte que quelques mois après le départ de Bonelli en Inde, celui-ci «écrivit une lettre à un des nôtres à Brera dans laquelle il décrivait tellement de maux corporels et spirituels du voyage en Inde et de la vocation d'y aller qu'elle dissuadait très efficacement d'entreprendre un tel voyage ${ }^{93}$. Recommandant le secret à son destinataire, le Père Sivori explique aussi que le contenu de la lettre ne fut pas divulgué ouvertement, mais qu'il fut néanmoins rapidement connu dans le collège sous forme de rumeurs. Selon lui, l'apparition de Cignardi n'a pas eu lieu à Brera mais pendant le voyage en Inde, alors que Bonelli était tenté de rentrer. Cela était pourtant "matériellement impossible", puisque Bonelli avait obtenu de partir comme missionnaire douze ans après la mort de son ami. Dans ce cas, la vision peut être considérée comme une grâce particulière puisque Bonelli aurait alors décidé de poursuivre son voyage.

Comment interpréter l'histoire elle-même, les différences constatées entre cette version et les trois autres et surtout son destin dans l'esprit de ceux qui restent? Que Cignardi soit apparu à Bonelli à Milan ou sur le bateau, l'apparition est un encouragement à poursuivre dans une vocation missionnaire partagée par les deux amis et dont on ne sait pas si elle surgit avant leur entrée dans l'ordre. Cette amitié qui, elle, précède l'entrée dans l'ordre, est fondatrice du caractère collectif de la vocation missionnaire pour tous ces jeunes jésuites. Cependant, la récupération de l'épisode dans divers récits et les différences de signification entre une apparition au moment même du décès de Cignardi face à son ami à peine éveillé et une vision du mort, douze ans après, sur le bateau menant Bonelli en Inde, montrent l'usage collectif de ce type de récit. Du point de vue de la tension dramatique et des conséquences collectives de l'épisode sur l'imaginaire des jeunes jésuites des collèges européens, il était beaucoup plus intéressant de le déplacer dans le temps et l'espace. Cela permet d'articuler plus facilement l'expérience singulière de l'apparition à l'histoire collective à laquelle participait tout jeune jésuite candidat à la mission. L'ambiguïté de Bonelli, que seule la transformation radicale de l'épisode par Sivori en 1668 permet de faire apparaître, révèle à la fois une faiblesse apparente et des sentiments contradictoires de peur et de désir auxquels les jeunes jésuites européens pouvaient tous s'identifier au moment de rédiger leur lettre Indipeta. La circulation clandestine du contenu de la lettre de Bonelli à Brera et le secret recommandé plus de quarante ans après dévoilent sa faiblesse mais invitent aussi au respect de la mémoire du missionnaire. S'il était honteux de vouloir rebrousser chemin une fois le pas du voyage franchi, personne n'était à l'abri de cette tentation. L'apparition d'un mort, ou plutôt la vision a posteriori d'un mort en odeur de sainteté, est d'une efficacité redoutable puisqu'elle redonne du courage au candidat missionnaire. Le récit 
reste donc dans la rhétorique propre aux récits de vie des missionnaires qu'on retrouve dans les Ménologes de l'ordre: une grande humilité, le doute éventuellement, surmonté néanmoins grâce à l'intervention de Dieu. Ce véritable exemplum montre la nécessité de dévoiler, littéralement de mettre à jour en permanence le désir de la mission, au sens du terme italien aggiornamento. Ce que dans leur répétition les lettres Indipetae font à l'échelle des individus, le récit d'une apparition y parvient collectivement, ce qui lui confère un statut de mythe particulièrement efficace dans le cadre de la solidarité générationnelle cimentée par la jeunesse et les liens amicaux.

Le rôle de la Compagnie de Jésus est bien sûr central dans la naissance de la vocation missionnaire des rédacteurs des lettres Indipetae. Pourtant, d'autres formes de sensibilisation à la mission existent dans le cadre de la vie laïque, au gré de lectures et de rencontres possibles dans la société européenne au début $\mathrm{du}$ XVII ${ }^{\mathrm{e}}$ siècle. À ces lectures s'ajoutent les liens entre milieux ecclésiastiques, milieux dévots et missions lointaines ${ }^{94}$. Cela traduit certainement le grand succès de la propagande de la Compagnie de Jésus pour la mission, au moins à l'échelle de la société urbaine milanaise. Les jeunes rédacteurs d'Indipetae, qui sont à la frontière du monde des laïcs et de celui des religieux, manifestent l'impact de cette propagande. Leurs lettres, et les épisodes qui entourent leur rédaction, en sont dans une certaine mesure le fruit, formulant à la fois la certitude de la nécessité de l'évangélisation et du départ en mission, mais aussi la peur et l'ignorance des réalités concrètes, que soulage la construction de véritables mythes.

Aliocha MALDAVSKY
UMR 8168 MASCIPO
Université Paris Ouest-Nanterre-La Défense
200 av. de la République
92001 Nanterre
aliocha.maldavsky@u-paris10.fr

94. Sans compter la prédication, les processions, le culte de saints missionnaires, également vecteurs de cette proximité, dans une perspective qui relève moins de la connaissance intellectuelle que de la sensibilité religieuse à la mission et qui mériterait des recherches approfondies. 